\title{
Synthesis parameter optimization for uniform and stable perovskite quantum dots
}

\author{
Dong Huang ${ }^{1}$, Ronghong Zheng ${ }^{1}$, Qinyi $\mathrm{Li}^{1}$, Chengzhao Luo ${ }^{1}$, and Yu Chen ${ }^{1,2, *}$ \\ ${ }^{1}$ School of Optoelectronic Science and Engineering, Soochow University, Suzhou 215006, P. R. China \\ ${ }^{2}$ National University of Singapore Suzhou Research Institute Dushu Lake Science and Education Innovation District Suzhou 215123, P. R. \\ China
}

\begin{abstract}
Colloidal lead halide perovskites nanocrystals, also known as quantum dots, have been intensively studied as promising optoelectronic materials and have attracted widespread attention due to their unique optical versatility, high photoluminescence quantum yield and convenient synthesis. Among them, the potential of all-inorganic halide caesium lead perovskite $\left(\mathrm{CsPbX}_{3}, \mathrm{X}=\mathrm{Cl}, \mathrm{Br}, \mathrm{I}\right)$ is particularly prominent. In this work, by adjusting the experimental parameters, including precursor preservation condition, reaction temperature and the isolation/purification process, $\mathrm{Cs}_{\mathrm{PbBr}}$ quantum dots with uniform size, neat arrangement, narrow full width at half maxima and excellent luminescent properties have been successfully prepared, which lays a good foundation for its potential application in practical optoelectronic devices.
\end{abstract}

\section{Introduction}

$\mathrm{CsPbBr}_{3}$ quantum dots (QDs) have broad application prospects in the fields of lighting and display due to their narrow emission line width $(12-42 \mathrm{~nm})$, high quantum yield (50-90\%) and short radiation lifetime (1-29 ns), and have attracted widespread attention in scientific field ${ }^{[1-4]}$.

Since Møller ${ }^{[5]}$ reported $\mathrm{CsPbX}_{3}(\mathrm{X}=\mathrm{Cl}, \mathrm{Br}$ or $\mathrm{I})$ perovskite in 1958 , the research on lead halide perovskite nanocrystals has made rapid progress in the past few decades. Protesescu ${ }^{[6]}$ et al. prepared high luminescent perovskite-based colloidal QDs and demonstrated a new way to prepare halide perovskites. They used cheaper materials and simple methods to synthesize monodisperse colloidal nanocubes of all-inorganic caesium halide lead perovskite with cubic shape and cubic perovskite crystal structure, which opened a new door for the preparation of lead halide perovskite nanocrystals.

However, the performance of $\mathrm{CsPbBr}_{3}$ QDs needs to be improved for practical applications. Therefore, meticulous preparation of QDs with multiple controllable parameters has become a top priority ${ }^{[7-10]}$. Herein, we successfully synthesized uniform and stable $\mathrm{CsPbr}_{3}$ QDs by thermal injection method. More importantly, by optimizing experimental parameters, including precursor preservation condition, reaction temperature and the isolation/purification process, the size distribution, colour purity, and storage stability of $\mathrm{CsPbrr}_{3}$ QDs have been significantly improved, which lays a foundation for its future application ${ }^{[11-13]}$.

\section{Materials and Methods}

\subsection{Materials}

Lead (II) bromide ( $\left.\mathrm{PbBr}_{2}, 99.99 \%\right)$, oleic acid (OA, 90\%), oleylamine (OAm, 90\%) and 1-octadecene (ODE, 90\%) were bought from Alfa Aesar. Caesium carbonate $\left(\mathrm{Cs}_{2} \mathrm{CO}_{3}\right.$, 99.99\%), methyl acetate (MAC, GR, 99\%), ethyl acetate (EAC, GR, 99.5\%), toluene (TL, GR, 99\%), tert-butanol (TBA, GR, 99\%) and n-hexane (Hex, GC, 99.0\%) were bought from Aladdin. All chemicals were used without purification unless otherwise noted.

\subsection{Synthetic methods}

\subsubsection{Preparation of Cs-Oleate Solution}

$\mathrm{Cs}_{2} \mathrm{CO}_{3}(407 \mathrm{mg}), 20 \mathrm{~mL}$ of ODE and $1.25 \mathrm{~mL}$ of OA were added to a $50 \mathrm{~mL}$ three-necked flask and maintained under vacuum at $120{ }^{\circ} \mathrm{C}$ for $60 \mathrm{~min}$. The mixture was stirred under $\mathrm{N}_{2}$ to $150{ }^{\circ} \mathrm{C}$ until all $\mathrm{Cs}_{2} \mathrm{CO}_{3}$ was dissolved. The solution was put aside for later use with the heating temperature being kept by various methods.

\subsubsection{Synthesis of $\mathrm{CsPbBr}_{3} \mathrm{QDs}$}

$\mathrm{PbBr}_{2}(414 \mathrm{mg})$ and $30 \mathrm{~mL}$ of ODE were placed in a 100 $\mathrm{mL}$ three-necked flask and maintained under vacuum at $120{ }^{\circ} \mathrm{C}$ for $60 \mathrm{~min}$. Then the reaction system was switched to nitrogen protection, $3 \mathrm{~mL}$ of OA and $3 \mathrm{~mL}$ of OAm were injected into the solution. Then the temperature was raised

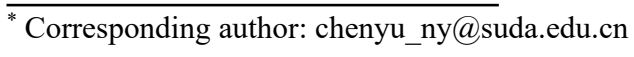


to $150{ }^{\circ} \mathrm{C}, 160{ }^{\circ} \mathrm{C}$ and $180{ }^{\circ} \mathrm{C}$, respectively, and the Cs-oleate was swiftly injected. After $5 \mathrm{~s}$, the reaction solution was put in an ice water bath and cooled to room temperature.

\section{Results and Discussion}

\subsection{The influence of precursor preservation condition on the morphology of $\mathrm{CsPbBr}_{3}$ QDs}

As mentioned in the synthetic method, the synthesis of $\mathrm{CsPbBr}_{3}$ QDs consisted of two steps, namely the precursor solution preparation followed by nucleation of the nanocrystals. The precursor solution played an important role in the whole experimental process, and its quality directly affected the results of the experiment.

In the preparation of precursor solution, $\mathrm{CsCO}_{3}$ was dissolved in organic solvent under high temperature. The obtained precursor solution was preserved in a vacuum oven at $120^{\circ} \mathrm{C}$, such method has been normally adopted in literature. However, after a period of time, the dissolved $\mathrm{CsCO}_{3}$ partially precipitated and caused the precursor solution to become turbid (Fig.1a). Therefore, we modified the precursor preservation condition by keeping it in an oil bath with inert atmosphere. As shown in Fig.1b, a clear and transparent precursor solution can be obtained.

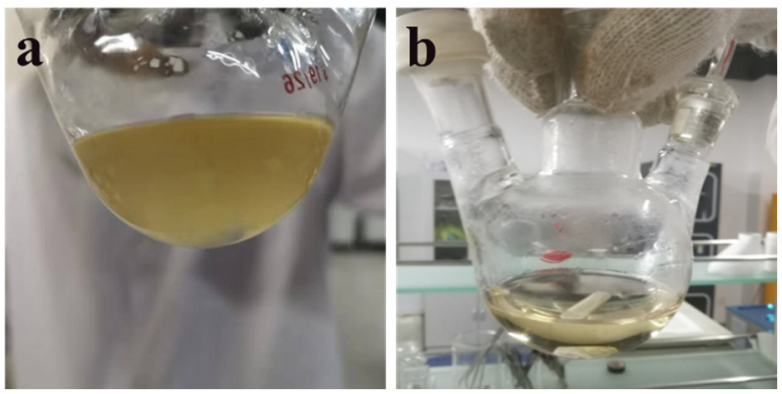

Fig. 1. Precursor solution after being preserved in (a) vacuum oven and (b) oil bath.

The different precursor preservation conditions had great influence on the resultant morphologies of obtained $\mathrm{CsPbr}_{3}$ QDs. TEM tests were carried out on the QDs prepared by the two thermal preservation methods. As shown in Fig.2 (a-c), QDs prepared by the precursor preserved in the vacuum oven was large in size and varied greatly in dimension. In comparison, as shown in Fig.2 (df), the QDs prepared by the precursor preserved in the oil bath are very uniform in size and neatly arranged. The average size of these QDs was about $8 \mathrm{~nm}$ as measured from TEM images.
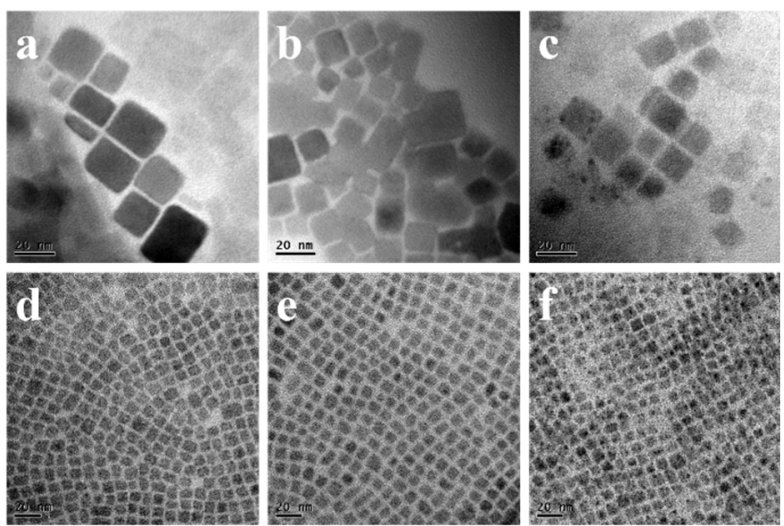

Fig. 2. TEM images of $\mathrm{CsPbBr}_{3}$ QDs prepared by using the precursor preserved in (a-c) vacuum oven. And (d-f) oil bath.

XRD measurement was carried out on the prepared QDs shown in Fig. 2(d-f) to examine their crystal structure in comparison with the standard PDF card. It can be obtained from Fig. 3 that the prepared QDs had a cubic crystal structure, and all diffraction peaks corresponded perfectly to the standard card (PDF\#54-0752) of $\mathrm{CsPbBr}_{3}$ QDs.

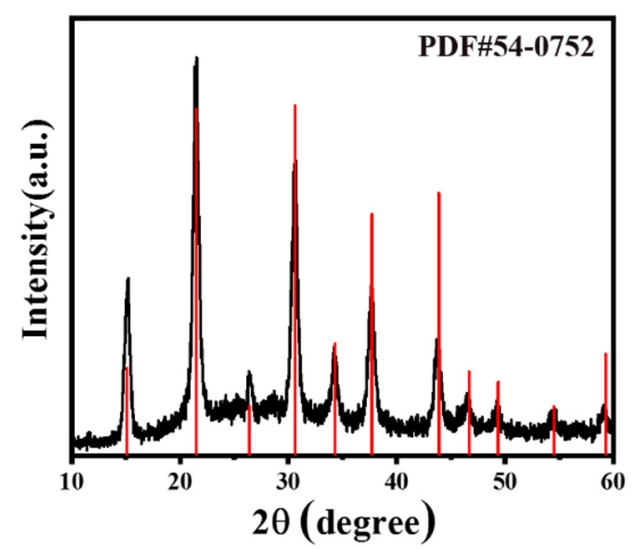

Fig. 3. XRD pattern of the prepared $\mathrm{CsPbBr}_{3}$ QDs.

\subsection{The influence of reaction temperature on the size of $\mathrm{CsPbBr}_{3}$ QDs}

The reaction temperature had a very crucial effect on the size of $\mathrm{CsPbBr}_{3}$ QDs, The average size of QDs have been successfully adjusted by adjusting the reaction temperature.

Fig.4 (a-c) shows the TEM images of $\mathrm{CsPbBr}_{3}$ QDs prepared at different temperatures of $150{ }^{\circ} \mathrm{C}, 160{ }^{\circ} \mathrm{C}$ and $180^{\circ} \mathrm{C}$. The average particle sizes of QDs prepared at 150 ${ }^{\circ} \mathrm{C}, 160{ }^{\circ} \mathrm{C}$ and $180{ }^{\circ} \mathrm{C}$ were measured to be $7.35 \mathrm{~nm}, 7.44$ $\mathrm{nm}$ and $8.15 \mathrm{~nm}$ respectively, with the size distributions shown in Fig. 4(d-f), indicating that in a certain range, the higher the preparation temperature, the larger the average particle size. 


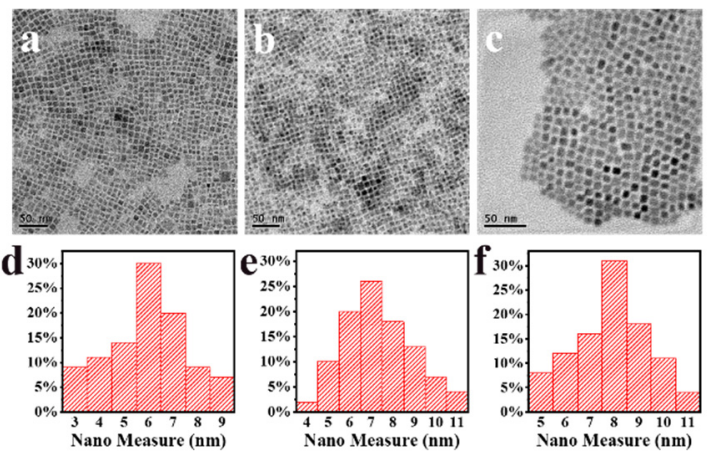

Fig. 4. TEM images of $\mathrm{CsPbBr}_{3}$ QDs prepared at (a) $150^{\circ} \mathrm{C}$, (b) $160^{\circ} \mathrm{C}$ and (c) $180^{\circ} \mathrm{C}$. Particle size distributions of $\mathrm{CsPbBr}_{3}$ QDs prepared at (d) $150{ }^{\circ} \mathrm{C}$, (e) $160^{\circ} \mathrm{C}$ and (f) $180^{\circ} \mathrm{C}$.

\subsection{The influence of isolation/purification on optical properties of $\mathrm{CsPbBr}_{3} \mathrm{QDs}$}

Isolation/purification of prepared QDs is a very important step that determines the dispersity and optical properties of QDs. During the isolation/purification of QDs, the excessive reagent as well as surface ligand were removed. Thus, the choice of solvents and washing times is the key to the long-term storage of QDs. Improper reagents will directly cause the agglomeration of QDs, and even destroy its structure, resulting in photoluminescence (PL) quenching ${ }^{[14]}$. So it is necessary to select appropriate reagents from a large number of attempts.

We divided the optimization of isolation/purification into three steps, namely choice of washing reagent, composition of washing reagent, and washing times. Four reagents were selected to purify QDs, namely TL, mixed solution of MAC and Hex, mixed solution of EAC and Hex, and mixed solution of TBA and Hex. PL test was carried out on the samples obtained by these four methods (Fig.5).
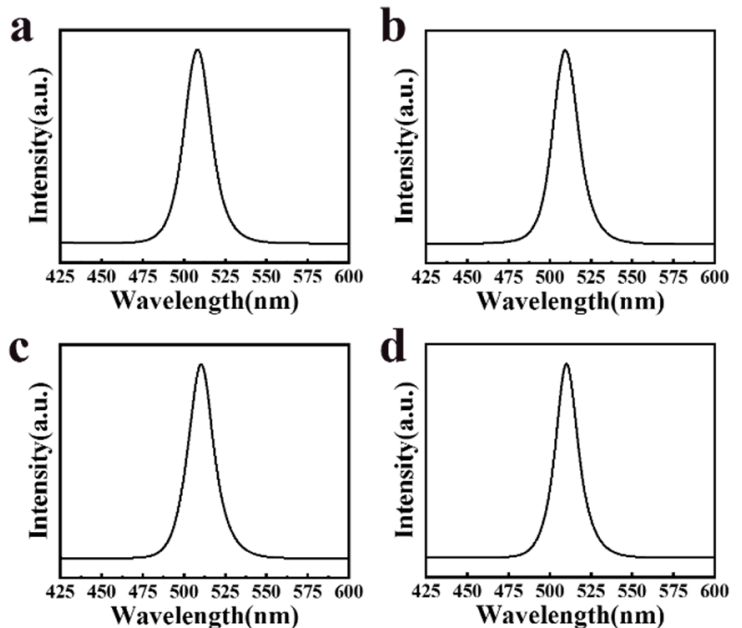

Fig. 5. $\mathrm{PL}$ patterns of $\mathrm{CsPbBr}_{3}$ QDs washed by (a) TL, (b) mixed solution of MAC and Hex, (c) mixed solution of EAC and Hex and (d) mixed solution of TBA and Hex.

The full width at half maxima (FWHM) of the four samples were measured and listed in Table 1. It can be seen that the FWHM of the QDs washed with TL was the largest, up to $23.5 \mathrm{~nm}$. The FWHM of the QDs washed by
MAC/Hex and EAC/Hex were smaller, being $21.5 \mathrm{~nm}$ and $20 \mathrm{~nm}$, respectively. And the FWHM of the QDs washed by mixed solution of TBA and TL was the smallest, being only $16 \mathrm{~nm}$. However, TBA would lead to easy agglomeration of QDs, thus reducing the optical performance and storage stability of QDs.

Table 1. PL analysis of QDs washed by different reagents.

\begin{tabular}{|c|c|c|c|c|}
\hline $\begin{array}{c}\text { Washing } \\
\text { method }\end{array}$ & TL & $\begin{array}{c}\text { MAC } \\
\text { and Hex }\end{array}$ & $\begin{array}{c}\text { EAC } \\
\text { and Hex }\end{array}$ & $\begin{array}{c}\text { TBA } \\
\text { and Hex }\end{array}$ \\
\hline $\begin{array}{c}\text { Emission } \\
\text { peak (nm) }\end{array}$ & 508 & 509.5 & 510.5 & 510 \\
\hline $\begin{array}{c}\text { FWHM } \\
(\mathrm{nm})\end{array}$ & 23.5 & 21.5 & 20 & 16 \\
\hline
\end{tabular}

In the previous attempt, the FWHM of the QDs washed by MAC/Hex and EAC/Hex were small, so we further adjusted the proportions of different reagents in the mixed solution. The PL spectra of QDs washed by the optimum reagent ratio of 3:1 are shown in Fig.6.
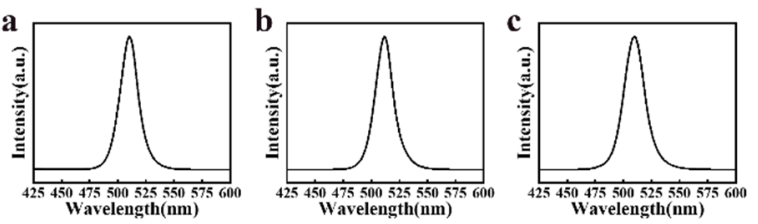

Fig. 6. $\mathrm{PL}$ patterns of $\mathrm{CsPbBr}_{3}$ QDs washed by (a) MAC: Hex=3:1, (b) EAC: Hex=3:1 and (c) Hex.

It can be seen from Table 2 that the FWHM of the QDs washed with EAC: Hex=3:1 is smallest, being $19 \mathrm{~nm}$, while those of the QDs washed with MAC: Hex=3:1 and Hex were larger, both of which being $21 \mathrm{~nm}$.

Table 2. PL analysis of QDs washed by different reagents.

\begin{tabular}{|c|c|c|c|}
\hline $\begin{array}{c}\text { Washing } \\
\text { method }\end{array}$ & $\begin{array}{c}\text { MAC: } \\
\text { Hex=3:1 }\end{array}$ & $\begin{array}{c}\text { EAC: } \\
\text { Hex=3:1 }\end{array}$ & Hex \\
\hline $\begin{array}{c}\text { Emission } \\
\text { peak (nm) }\end{array}$ & 510 & 511.5 & 510 \\
\hline $\begin{array}{c}\text { FWHM } \\
(\mathrm{nm})\end{array}$ & 21 & 19 & 21 \\
\hline
\end{tabular}

Finally, the influence of washing times on QDs was also analysed. By fixing the washing reagent of EAC: Hex=3:1, QDs were washed by different times and then analysed by PL test (Fig.7).
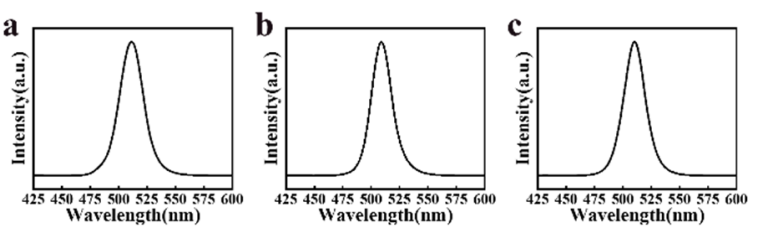

Fig. 7. $\mathrm{PL}$ patterns of $\mathrm{CsPbBr}_{3}$ QDs washed with EAC: Hex $=3: 1$ for (a) once, (b) twice and (c) three times.

As shown in Table 3, the FWHM of the QDs obtained after twice washing was $18.5 \mathrm{~nm}$, which is significantly smaller than those of QDs washed once and three times. The smaller the FWHM, the higher the luminous purity of 
QDs. Therefore, the optimized washing parameters of QDs was determined to be washed twice with EAC: Hex $=3: 1$.

Table 3. Effect of washing times with EAC: $H e x=3: 1$ on QDs.

\begin{tabular}{|c|c|c|c|}
\hline $\begin{array}{c}\text { Washing } \\
\text { times }\end{array}$ & 1 & 2 & 3 \\
\hline $\begin{array}{c}\text { Emission } \\
\text { peak (nm) }\end{array}$ & 511.5 & 509 & 510 \\
\hline $\begin{array}{c}\text { FWHM } \\
(\mathrm{nm})\end{array}$ & 20 & 18.5 & 19.5 \\
\hline
\end{tabular}

$\mathrm{CsPbBr}_{3}$ QDs can be greatly affected by the polarity of organic solvents. Solvent with strong polarity would have a significant negative impact on the optical property and structure intergrity of QDs. Therefore, Hex with less polarity was used as the solvent of $\mathrm{CsPbBr}_{3}$ QDs.

It can be seen from Fig.8, after adjusting the experimental parameters, the synthesized $\mathrm{CsPbBr}_{3}$ QDs was a clear and transparent light green solution, which emits green fluorescence under the irradiation of ultraviolet (UV) lamp (365nm).
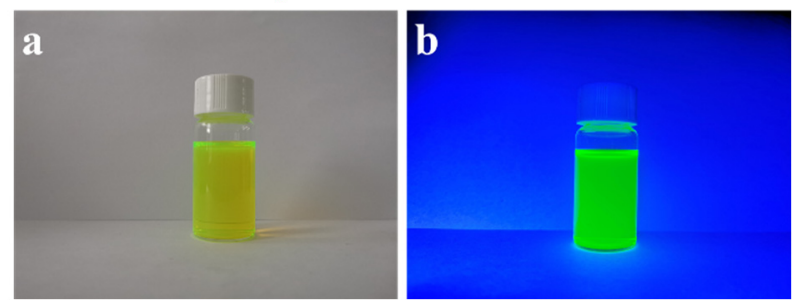

Fig. 8. Photographs of the $\mathrm{CsPbBr}_{3}$ QDs solution under (a) natural light and (b) UV light (365 nm)

\subsection{Research on storage stability of $\mathrm{CsPbBr}_{3} \mathrm{QDs}$}

$\mathrm{CsPbBr}_{3}$ QDs are very fragile and may lose their structure integrity and optical properties by a variety of external stimuli, including light, heat, and oxygen, which is a major limiting factor for its practical application ${ }^{[15,16]}$.

The prepared $\mathrm{CsPbBr}_{3}$ QDs were stored in a fridge frozen chamber, and taken out for PL and UV tests every other week. It can be seen from Fig.9 that after nearly a month of storage, the PL and UV curves of QDs were basically unchanged, being highly coincident with the beginning curve, showing good storage stability of $\mathrm{CsPbBr}_{3}$ QDs prepared by optimized method.
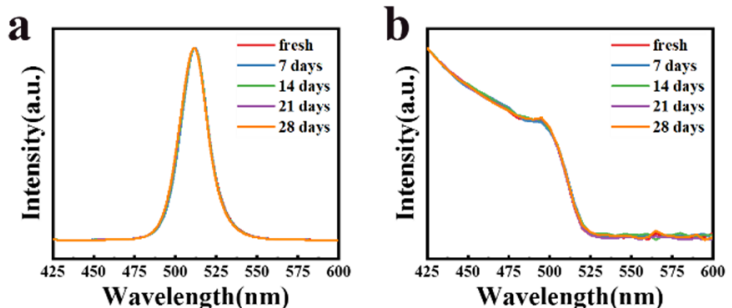

Fig. 9. (a) $\mathrm{PL}$ and (b) UV spectra of $\mathrm{CsPbBr}_{3}$ QDs after various storage periods up to 28 days.

\section{Conclusion}

In conclusion, we demonstrate a systematic analysis on the synthesis parameters of $\mathrm{CsPbBr}_{3}$ QDs by adjusting precursor preservation condition, reaction temperature and isolation/purification process. Compared with the previously reported $\mathrm{CsPbBr}_{3}$ QDs, the QDs synthesized in this work show a high degree of monodispersity and narrow FWHM. More importantly, satisfactory storage stability has been demonstrated, which is of great significance for the potential practical application of perovskite QDs.

\section{Acknowledgments}

This work was supported by the Natural Science Foundation of Jiangsu Province of China (Grant Nos. BK20190098), the Natural Science Foundation of the Jiangsu Higher Education Institutions of China (19KJA550001), Key Lab of Modern Optical Technologies of Education Ministry of China, Key Lab of Advanced Optical Manufacturing Technologies of Jiangsu Province, Priority Academic Program Development (PAPD) of Jiangsu Higher Education Institutions, and Jiangsu Shuangchuang Plan.

\section{References}

1. Y. Wang, X. Li, J. Song, L. Xiao, H. Zeng, H. Sun, Adv. Mater. 2015, 27, 7101-7108

2. A. S. M. Chong, X. S. Zhao, J. Phys. Chem. B 2003, 107, 12650-12657.

3. L. Zhang, X. Sun, Y. Song, X. Jiang,S. Dong, E. Wang, Langmuir 2006, 22, 2838-2843.

4. S. Jun, J. Lee, E. Jang, ACS Nano 2013, 7, 1472-1477.

5. C. K. Møller, Nature 1958, 182, 1436 - 1436.

6. G. Nedelcu, L. Protesescu, S. Yakunin, M. I. Bodnarchuk, M. J. Grotevent, M. V. Kovalenko, Nano Lett. 2015, 15, 5635-5640

7. W. Chen, J. Hao, W. Hu, Z. Zang, X. Tang, L. Fang, T. Niu, M. Zhou, Small 2017, 13, 1604085

8. F. Palazon, Q. A. Akkerman, M. Prato, L. Manna, ACS Nano 2016, 10, 1224-1230.

9. K. Xu, C. C. Lin, X. Xie, A. Meijerink, Chem. Mater. 2017, 29, 4265-4272

10. G. Yang, Q. Fan, B. Chen, Q. Zhou, H. Zhong, J. Mater. Chem. C 2016, 4, 11387-11391.

11. A. M. Munro, I. J.-L. Plante, M. S. Ng, D. S. Ginger, J. Phys. Chem. C 2007, 111, 6220-6227

12. D.-K. Ko, A. Maurano, S. K. Suh, D. Kim, G. W. Hwang, J. C. Grossman, V. Bulovic ', M. G. Bawendi, ACS Nano 2016, 10, 3382-3388

13. J. Pan, S. P. Sarmah, B. Murali, I. Dursun, W. Peng, M. R. Parida, J. Liu, L. Sinatra, N. Alyami, C. Zhao, E. Alarousu, T. K. Ng, B. S. Ooi, O. M. Bakr, O. F. Mohammed, J. Phys. Chem. Lett. 2015, 6, 5027-5033

14. F. Di Stasio, S. Christodoulou, N. Huo, G. 
Konstantatos, Chem. Mater. 2017, 29, 7663.

15. S. Wei, Y . Y ang, X. Kang, L. Wang, L. Huang, D. Pan, Inorg. Chem. 2017, 56, 2596.

16. H. Huang, F. Zhao, L. Liu, F. Zhang, X.-g. Wu, L. Shi, B. Zou, Q. Pei, H. Zhong, ACS Appl. Mater. Interfaces 2015, 7, 28128. 